12

\title{
Высокодисперсионные электростатические зеркала вращательной симметрии с времяпролетной фокусировкой по энергии третьего порядка
}

\author{
(C) С.Б. Бимурзаев, Н.У. Алдияров, З.С. Саутбекова
}

Алматинский университет энергетики и связи, Алматы, Казахстан

e-mail: bimurzaev@mail.ru

Поступило в Редакцию 25 июня 2019 г.

В окончательной редакции 10 декабря 2019 г.

Принято к публикации 27 января 2020 г.

\begin{abstract}
Приведены выражения, описывающие время пролета заряженных частиц с учетом времяпролетных хроматических аберраций в электростатических зеркалах вращательной симметрии. Путем численных расчетов найдены условия времяпролетной фокусировки по энергии частиц до третьего порядка включительно в трехэлектродных зеркалах, электродами которых служат соосные цилиндры равного диаметра. Рассмотрены различные режимы работы таких зеркал: 1) пространственно-времяпролетная фокусировка в гауссовой плоскости зеркала; 2) пространственно-времяпролетная фокусировка в фокальной плоскости зеркала; 3) времяпролетная фокусировка в телескопическом режиме; 4) времяпролетная фокусировка в коллиматорном режиме. Результаты расчета представлены в зависимости от двух фундаментальных решений уравнения параксиальных траекторий. Одно из этих решений описывает траектории, прямая и обратная ветви которых совпадают, а второе решение - траектории, прямая и обратная ветви которых симметричны относительно оси симметрии поля. Показано, что времяпролетная дисперсия зеркала по массам, определенная на основе второго решения, в несколько раз выше, чем на основе первого решения для всех режимов его работы.
\end{abstract}

Ключевые слова: времяпролетный масс-рефлектрон, времяпролетная дисперсия по массе, времяпролетная хроматическая аберрация, времяпролетная фокусировка, эффективное дрейфовое расстояние.

DOI: 10.21883/JTF.2020.07.49456.280-19

\section{Введение}

Разрешающая способность времяпролетного массрефлектрона (ВПМР) увеличивается пропорционально времени пролета ионов от источника до детектора [1], что связано с уменьшением относительного вклада в разрешающую способность временно́го разброса ионов в пакете, формируемом в ионном источнике. Увеличить время пролета ионов (т. е. времяпролетную дисперсию по массам) в ВПМР можно двумя способами: 1) наращиванием длины пролета ионов от источника до детектора; 2) путем определенного подбора распределения поля в электростатическом зеркале, выполняющем роль ионного рефлектора.

Первый способ увеличения времени пролета ионов реализован в многоотражательных ВПМР, в которых роль ионного рефлектора выполняет система, состоящая из двух параллельных друг другу двумерных электростатических зеркал [2,3]. При этом предельное разрешение многоотражательного ВПМР остается пропорциональным времени пролета ионов при однократном отражении зеркалом [3].

Второй способ увеличения времени пролета ионов описан в работе [4], в которой впервые было показано, что в двухэлектродном электростатическом зеркале вращательной симметрии могут быть реализованы два случая пространственно- времяпролетной фокусировки первого порядка. В первом случае узловая плоскость времяпролетной фокусировки совмещена с плоскостью, проходящей через центр кривизны зеркала, а во втором - через его вершину. При этом время пролета ионов в зеркале, следовательно, эффективное дрейфовое расстояние зеркала во втором случае значительно больше, чем в первом.

Несмотря на это, зеркала с режимом фокусировки с большим временем пролета практически были преданы забвению как менее светосильные, так как с увеличением длины пробега частиц происходит естественное уменьшение апертурных углов. При этом из поля зрения выпала другая причина, влияющая на светосилу зеркала - его пропускная способность. Вместе с тем, как показано в работе [5], пропускная способность двухэлектродного зеркала вращательной симметрии со вторым режимом фокусировки значительно больше по сравнению с первым режимом фокусировки.

В [6] были исследованы свойства времяпролетной фокусировки двухэлектродного зеркала вращательной симметрии для двух режимов его работы, когда светосила зеркала не зависит от длины пробега частиц: 1) телескопический режим - падающий на зеркало параллельный пучок частиц остается параллельным и после отражения зеркалом; 2) режим параллель/точка - 
падающий на зеркало параллельный пучок частиц фокусируется в фокальной плоскости зеркала. Результаты этих исследований показывают, что в двухэлектродном зеркале вращательной симметрии может быть достигнута времяпролетная фокусировка частиц по энергии при высокой времяпролетной дисперсии зеркала по массам как в телескопическом режиме, так и в режиме параллель/точка. Однако в двухэлектродном зеркале невозможна времяпролетная фокусировка по энергии выше первого порядка, что ограничивает их практическое применение для повышения разрешающей способности ВПМР. Это приводит к необходимости более детального изучения фокусирующих свойств высокодисперсионных зеркал вращательной симметрии на основе многоэлектродных систем, имеющих большее разнообразие в распределении поля.

В настоящей работе путем численных расчетов исследованы дисперсионные свойства трехэлектродных электростатических зеркал вращательной симметрии для различных режимов их работы при выполнении условий времяпролетной фокусировки по энергии до третьего порядка включительно.

\section{1. Расчетные формулы для исследования электростатических зеркал}

\section{1. Время пролета}

В задачах времяпролетной фокусировки пучков заряженных частиц особый интерес представляют времяпролетные хроматические аберрации (ВПХА). Это связано с тем, что в электронно-оптических системах с прямой оптической осью времяпролетные геометрические аберрации эффективно уменьшаются простым диафрагмированием этих пучков. ВПХА при этом остаются без изменений и накладывают принципиальные ограничения на качество времяпролетной фокусировки. По этой причине в дальнейшем ограничимся рассмотрением этих аберраций.

Для изучения времяпролетных хроматических аберраций зеркала достаточно рассмотреть движение частиц вдоль его главной оптической оси и определить зависимость времени пролета частиц от их начальных энергий.

Введем цилиндрическую систему координат $r, \psi, z$, в которой ось $z$ совмещена с главной оптической осью зеркала. Согласно [7], время пролета иона заряда $q$ и массы $m$, движущегося вдоль оптической оси зеркала $z$ от начальной плоскости $z=z_{0}$ до произвольной плоскости $z=$ const, с учетом величин до третьего порядка малости может быть записано в виде

$$
t=T+\Delta t
$$

Здесь

$$
T=-\frac{1}{v_{0}}\left(z+z_{0}-2 z_{T}^{(0)}\right)
$$

- время пролета центрального (движущегося вдоль оси $z$ с нулевым разбросом энергий) иона,

$$
\Delta t=\frac{1}{v_{0}} \sum_{k=1}^{3}\left(\frac{\varepsilon}{\Phi_{0}}\right)^{k} D_{t \varepsilon}^{(k)}
$$

- суммарная ВПХА зеркала, $\varepsilon$ - начальный разброс энергий ионов, $\Phi=\Phi(z)$ - осевое распределение электростатического потенциала, $v_{0}=\sqrt{-2 q \Phi / m}-$ скорость центрального иона, а

$$
D_{t \varepsilon}^{(k)}=(-1)^{k+1} \frac{(2 k-1) ! !}{(2 k) ! !}\left(z+z_{0}-2 z_{T}^{(k)}\right) \quad(k=1,2,3)
$$

- коэффициент ВПХА $k$-го порядка. Величины $z_{T}^{(0)}$ и $z_{T}^{(k)}(k=1,2,3)$, определяющие, как это видно из (2) и (4), положения эффективной плоскости отражения зеркала и его плоскостей времяпролетной фокусировки $k$-го порядка соответственно, являются функциями лишь осевого распределения потенциала $\Phi=\Phi(z)$ зеркала [7].

Здесь и далее нижним индексом „0“ отмечены значения величин в начальной (предметной) плоскости $z=z_{0}$

\section{2. Условия времяпролетной фокусировки}

Как видно из (4), условие времяпролетной фокусировки ионов по энергии $k$-го порядка $\left(D_{t \varepsilon}^{(k)}=0\right)$ определяется равенством

$$
z+z_{0}=2 z_{T}^{(k)}
$$

которое означает, что времяпролетная фокусировка достигается, если плоскости $z=z_{0}$ (предметная плоскость) и $z=$ const (плоскость времяпролетного изображения) расположены симметрично относительно плоскости $z=z_{T}^{(k)}(k=1,2,3)$.

При выполнении условия

$$
z_{T}=z_{T}^{(1)}=z_{T}^{(2)}=z_{T}^{(3)}
$$

одновременно с (5) все коэффициенты ВПХА равны нулю $\left(D_{t \varepsilon}^{(1)}=D_{t \varepsilon}^{(2)}=D_{t \varepsilon}^{(3)}=0\right)$, т.е. в плоскости $z=z_{T}$ реализуется времяпролетная фокусировка ионов по энергии до третьего порядка включительно.

Плоскость $z=z_{T}^{(1)}$ называют главной плоскостью времяпролетной фокусировки зеркала [4]. С учетом (5) можно записать (2) в виде

$$
T=\frac{2}{v_{0}}\left(z_{T}^{(0)}-z_{T}^{(1)}\right) .
$$

Равенство (7) определяет интервал времени между моментами пересечения плоскости $z=z_{T}^{(1)}$ ионами до 
и после отражения зеркалом. Этот интервал времени называют временным интервалом фокусировки зеркала. Зависимость временного интервала фокусировки от массы определяет величину его времяпролетной дисперсии по массам [4]

$$
D_{t m}=m \frac{\partial T}{\partial m}=\frac{T}{2}=\frac{L}{v_{0}}
$$

где

$$
L=\frac{1}{2} v_{0} T
$$

- эффективное дрейфовое расстояние зеркала.

Как известно, предельная разрешающая способность масс-рефлектрона с электростатическим зеркалом равна

$$
R=\frac{T}{2 \Delta t_{i}}
$$

где $\Delta t_{i}$ - временно́й разброс пакета ионов в первичном временно́м фокусе.

С учетом равенств (8), (9) можно записать равенство (10) в виде

$$
R=\frac{D_{t m}}{\Delta t_{i}}=\frac{L}{\Delta z_{i}}
$$

где $\Delta z_{i}=v_{0} \Delta t_{i}$ - ширина пакета ионов в первичном временно́м фокусе.

\section{3. Уравнения траекторий}

Согласно [7], уравнения параксиальной траектории и ее наклона к оптической оси $z$ для частицы, движущейся от начальной плоскости $z=z_{0}$ до произвольной плоскости $z=$ const (после отражения зеркалом), можно записать в виде

$$
\begin{gathered}
r=-\frac{2 \sqrt{\Phi_{0}}}{\Phi_{u}^{\prime}}\left[r_{0}^{\prime}\left(p_{0} g+p g_{0}\right)-r_{0}\left(p_{0}^{\prime} g+p g_{0}^{\prime}\right)\right], \\
r^{\prime}=-\frac{2 \sqrt{\Phi_{0}}}{\Phi_{u}^{\prime}}\left[r_{0}^{\prime}\left(p_{0} g^{\prime}+p^{\prime} g_{0}\right)-r_{0}\left(p_{0}^{\prime} g^{\prime}+p^{\prime} g_{0}^{\prime}\right)\right] .
\end{gathered}
$$

Здесь $p=p(z)$ и $g=g(z)$ - линейно независимые частные решения параксиального уравнения

$$
\Phi r^{\prime \prime}+\frac{1}{2} \Phi^{\prime} r^{\prime}+\frac{1}{4} \Phi^{\prime \prime} r=0
$$

При этом $p=p(z)$ является аналитической функцией, a $g=g(z)$ может быть представлено в виде

$$
g=\sqrt{\Phi} q
$$

где $q=q(z)$ - аналитическая функция, удовлетворяющая уравнению

$$
\Phi q^{\prime \prime}+\frac{3}{2} \Phi^{\prime} q^{\prime}+\frac{3}{4} \Phi^{\prime \prime} q=0
$$

следующему из (14) и (15).
Функции $p=p(z)$ и $q=q(z)$ удовлетворяют следующим начальным условиям:

$$
p_{u}=q_{u}=1, \quad p_{u}^{\prime}=q_{u}^{\prime}=-\frac{\Phi_{u}^{\prime \prime}}{2 \Phi_{u}^{\prime}} .
$$

Здесь и далее штрихи обозначают дифференцирование

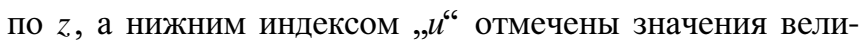
чин в плоскости поворота ионов $z=z_{u}$ зеркала.

\section{4. Режимы работы зеркала}

Как видно из (12), положение гауссовой плоскости изображения $z=z_{G}$ (условие стигматичной пространственной фокусировки - режим точка в точку) определяется равенством

$$
p_{0} g\left(z_{G}\right)+p\left(z_{G}\right) g_{0}=0 .
$$

Откуда с учетом (12) получим

$$
\frac{p\left(z_{G}\right)}{p_{0}}=-\frac{g\left(z_{G}\right)}{g_{0}}=\frac{r}{r_{0}}=M,
$$

где $M$ - линейное увеличение зеркала.

Положение фокальной плоскости $z=z_{F}$ (условие фокусировки параллельных пучков частиц - режим параллель/точка), как это видно из (12), определяется равенством

$$
p_{0}^{\prime} g\left(z_{F}\right)+p\left(z_{F}\right) g_{0}^{\prime}=0 .
$$

Откуда с учетом (12) следует

$$
\frac{p\left(z_{F}\right)}{p_{0}^{\prime}}=-\frac{g\left(z_{F}\right)}{g_{0}^{\prime}}=\frac{r}{r_{0}^{\prime}}=f_{0},
$$

где $f_{0}$ - фокусное расстояние предметного пространства зеркала.

Коллиматорный режим работы зеркала (условие параллельности в пространстве изображений ионного пучка, исходящего из точечного источника $\left(r_{0}=0\right)-$ режим точка/параллель) определяется, как это видно из (13), равенством

$$
p_{0} g^{\prime}+g_{0} p^{\prime}=0
$$

С учетом (13) из (22) следует, что

$$
\frac{p_{0}}{p^{\prime}}=-\frac{g_{0}}{g^{\prime}}=\frac{r_{0}}{r^{\prime}}=f,
$$

где $f-$ фокусное расстояние пространства изображений, которое связано с фокусным расстоянием предметного пространства $f_{0}$ соотношением

$$
f=f_{0},
$$

следующим из (12) и (13).

Телескопический режим (падающий на зеркало параллельный пучок остается параллельным и после отражения), как это видно из (13), определяется равенством

$$
p_{0}^{\prime} g^{\prime}+g_{0}^{\prime} p^{\prime}=0 \text {. }
$$




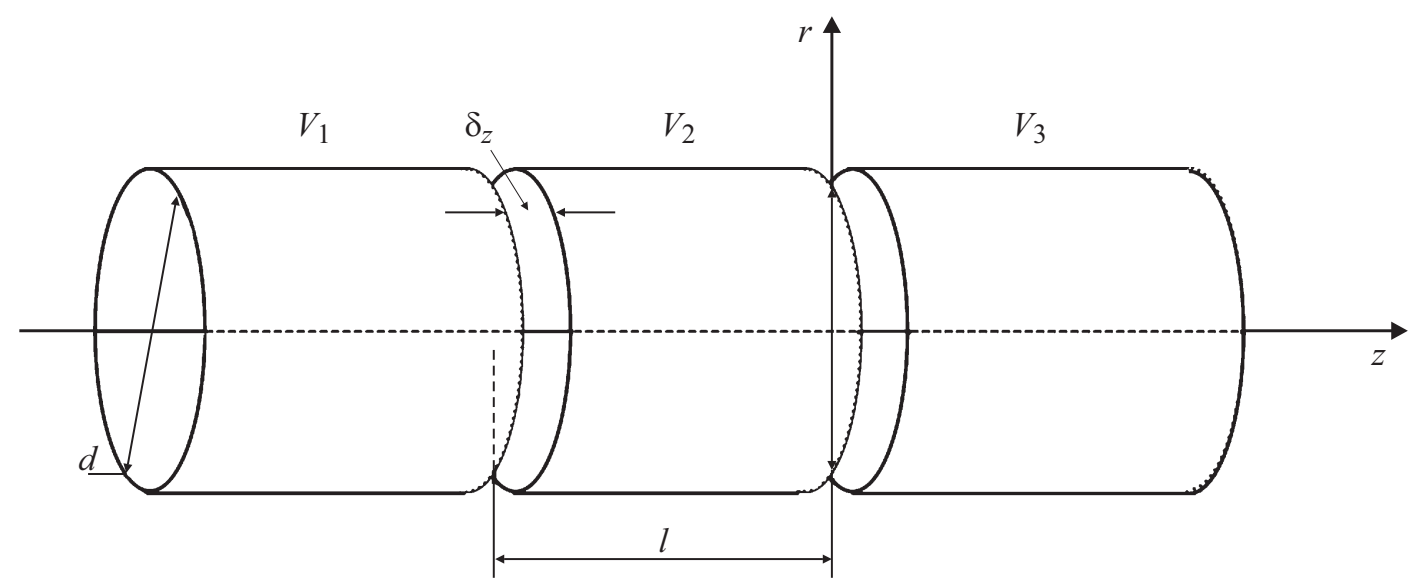

Рис. 1. Трехэлектродное зеркало вращательной симметрии: $V_{1}, V_{2}, V_{3}-$ потенциалы на электродах, $d-$ диаметр цилиндра, $l$ - длина среднего электрода, $\delta_{z}$ - ширина зазора между электродами.

При этом, как следует из (13) и (25):

$$
\frac{p^{\prime}}{p_{0}^{\prime}}=-\frac{g^{\prime}}{g_{0}^{\prime}}=\frac{r^{\prime}}{r_{0}^{\prime}}=\gamma,
$$

где $\gamma$ - угловое увеличение зеркала, которое связано с его линейным увеличением $M$ соотношением

$$
M \gamma=-1
$$

следующим из (12) и (13).

Определим кардинальные элементы электронного зеркала. Для этого воспользуемся характерными для зеркала траекториями. Решение $p=p(z)$ описывает траектории, прямые и обратные ветви которых совпадают, т.е. проходят через центр кривизны зеркала $z=z_{C}$, а решение $g=g(z)$ - траектории, прямые и обратные ветви которых симметричны относительно оптической оси зеркала, т.е. проходят через вершину зеркала $z=z_{V}$ [4]. Таким образом, когда предмет и его изображение находятся в свободном от поля пространстве, функции $p=p(z)$ и $g=g(z)$ можно записать так

$$
p=\left(z-z_{C}\right) p^{\prime}, \quad g=\left(z-z_{V}\right) g^{\prime} .
$$

С учетом этих равенств из (20)-(21) следует, что положение фокуса зеркала и его фокусное определяются равенствами

$$
z_{F}=\frac{1}{2}\left(z_{V}+z_{C}\right), \quad f=\frac{1}{2}\left(z_{V}-z_{C}\right) .
$$

Как следует из (22), коллиматорный режим зеркала реализуется при выполнении очевидного условия

$$
z_{0}=z_{F}
$$

В свободном от поля пространстве телескопический режим зеркала определяется равенством

$$
p^{\prime}=0 \quad \text { или } \quad g^{\prime}=0 .
$$

Решая уравнение (18) с учетом (28), (29), можно записать уравнение пространственных изображений для зеркала в виде „уравнений Ньютона“

$$
\left(z_{0}-z_{F}\right)\left(z_{G}-z_{F}\right)=f^{2} .
$$

При этом линейное увеличение зеркала равно

$$
M=\frac{f}{z_{0}-z_{F}}=\frac{z_{G}-z_{F}}{f} .
$$

\section{5. Условия пространственно-времяпролетной фокусировки}

Решая систему уравнений (4) и (32) с учетом (5), (6), получим соотношение

$$
z_{1,2}=z_{T} \pm \sqrt{\left(z_{T}-z_{C}\right)\left(z_{T}-z_{V}\right)}
$$

определяющее положения взаимосопряженных плоскостей $z=z_{1}$ и $z=z_{2}$, для которых осуществляется пространственная и времяпролетная фокусировка одновременно.

При выполнении условия

$$
z_{T}-z_{C} \text { или } z_{T}-z_{V},
$$

из (34) следует, что

$$
z_{1}=z_{2}=z_{T}
$$

т. е. пространственно-времяпролетная фокусировка осуществляется в одной и той же плоскости $z=z_{T}$.

\section{2. Расчет трехэлектродного зеркала вращательной симметрии}

Рассматриваемое зеркало (рис. 1) состоит из трех соосных цилиндров равного диаметра $d$, находящихся под потенциалами $V_{1}, V_{2}, V_{3}$. 
Таблица 1. Характеристики трехэлектродного зеркала вращательной симметрии, обусловленные $p$-решением

\begin{tabular}{c|c|c|c|c|c}
\hline Режим & $V_{2} / V_{1}$ & $-V_{3} / V_{2}$ & $l / d$ & $-z_{T} / d$ & $L / d$ \\
\hline$p^{\prime}=0$ & 0.0539 & 7.87 & 0.632 & 4.65 & 5.50 \\
\hline$z_{F}=z_{T}$ & 0.0479 & 7.90 & 0.650 & 5.44 & 6.36 \\
\hline$z_{C}=z_{T}$ & 0.0432 & 7.92 & 0.666 & 6.23 & 7.22
\end{tabular}

Таблица 2. Характеристики трехэлектродного зеркала вращательной симметрии, обусловленные $g$-решением

\begin{tabular}{c|c|c|c|c|c}
\hline Режим & $V_{2} / V_{1}$ & $-V_{3} / V_{2}$ & $l / d$ & $-z_{T} / d$ & $L / d$ \\
\hline$g^{\prime}=0$ & 0.00932 & 8.45 & 0.932 & 51.7 & 54.4 \\
\hline$z_{F}=z_{T}$ & 0.00919 & 8.46 & 0.935 & 52.7 & 55.4 \\
\hline$z_{V}=z_{T}$ & 0.00907 & 8.47 & 0.937 & 53.8 & 56.5
\end{tabular}

Осевое распределение электростатического потенциала зеркала описывается соотношением [8]

$$
\Phi(z)=\frac{1}{2}\left[\left(V_{1}+V_{3}\right)+\sum_{i=1}^{2}\left(V_{i+1}-V_{i}\right) U\left(z-z_{i}\right)\right],
$$

где

$$
\begin{aligned}
& U\left(z-z_{i}\right)=\operatorname{sign}\left(z-z_{i}\right) \\
& \times\left[1-\sum_{s=1}^{\infty} \exp \left(-2 \alpha_{s}\left|z-z_{i}\right| / d\right) / \prod_{m=1}^{\infty}\left(1-\alpha_{s}^{2} / \alpha_{m}^{2}\right)_{s \neq m}\right] .
\end{aligned}
$$

Здесь $z_{i}$ - координата середины $i$-го зазора между электродами, $\alpha_{s}, \alpha_{m}-$ корни функции Бесселя нулевого порядка. Принято, что положительное направление оси $z$ совпадает с направлением движения падающих на зеркало ионов, а начало координат помещено в середине второго зазора (между вторым и третьим электродами).
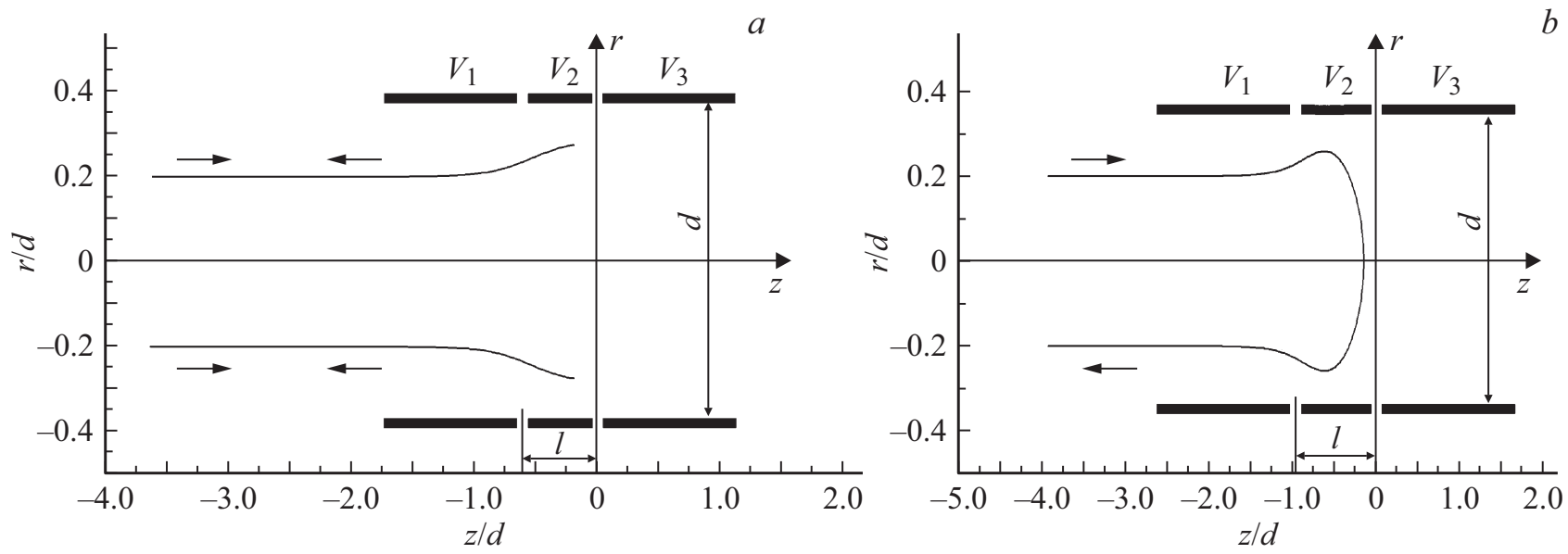

Pис. 2. Ход траекторий частиц в телескопическом режиме: $a-p^{\prime}=0 ; b-g^{\prime}=0$.
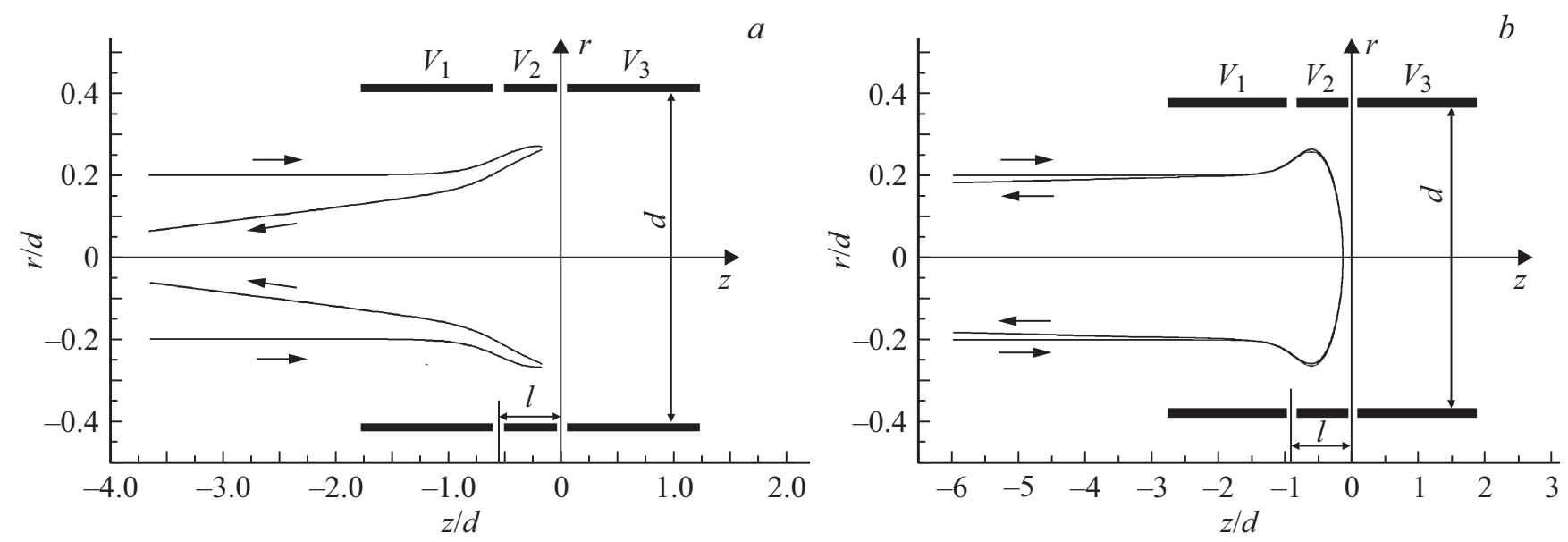

Рис. 3. Ход траекторий частиц в режиме параллель/точка $\left(z_{T}=z_{F}\right): a-p$-решение; $b-g$-решение. 

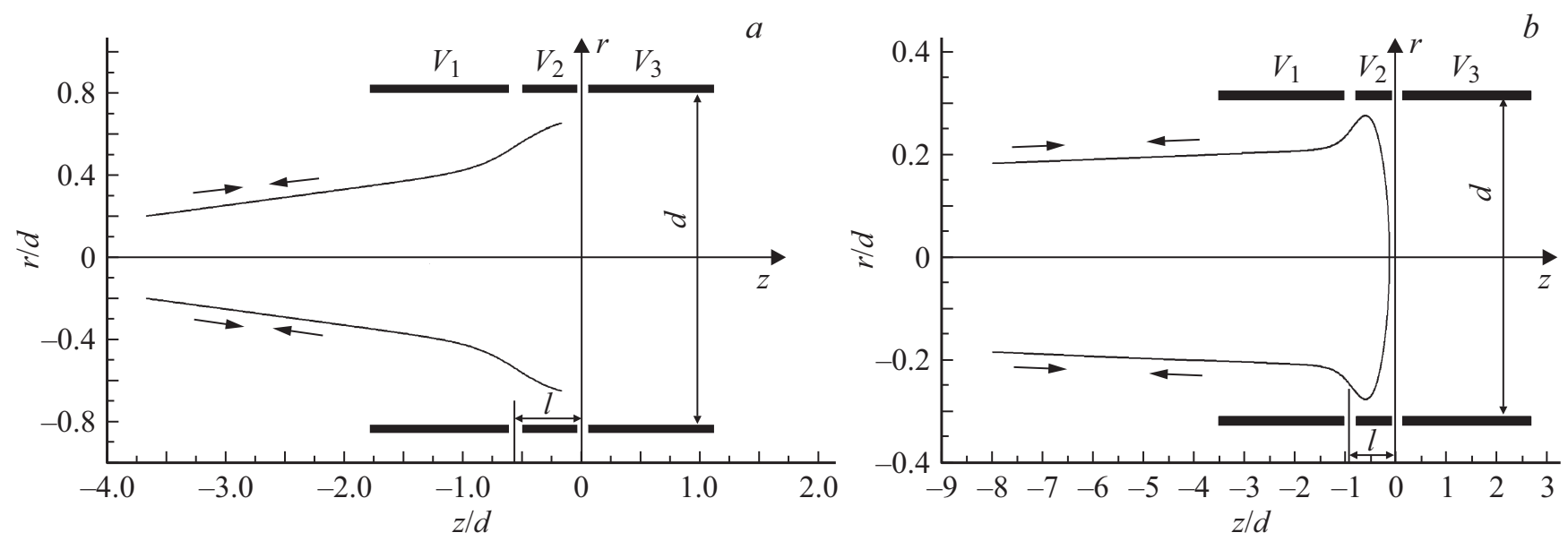

Pис. 4. Ход траекторий частиц в режиме точка в точку: $a-z_{T}=z_{C} ; b-z_{T}=z_{V}$.

Расчет зеркала производился следующим образом. Для заданных значений потенциала $V_{2}$ на втором (среднем) электроде и ширины $l$ среднего электрода находились значения запирающего потенциала $V_{3}$ на третьем электроде, обеспечивающие выполнение условий времяпролетной фокусировки по энергии до третьего порядка включительно (6) для различных режимов работы зеркала.

В табл. 1 и 2 представлены соотношения геометрических и электрических параметров, определяющие различные режимы работы зеркала: 1) телескопический режим $\left(p^{\prime}=0\right.$ или $\left.g^{\prime}=0\right)$; 2) режим параллель/точка $\left.\left(z_{F}=z_{T}\right) ; 3\right)$ режим точка в точку $\left(z_{C}=z_{T}\right.$ или $\left.z_{V}=z_{T}\right)$.

Заметим, что в силу обратимости хода траекторий в зеркале коллиматорный режим реализуется при тех же условиях, что и режим параллель/точка.

В табл. 1 приведены результаты расчета, обусловленные $p$-решением, а в табл. 2 - результаты расчета, обусловленные $g$-решением.

Как видно из приведенных в таблицах данных, значения удельного эффективного дрейфового расстояния $L / d$ (т.е. эффективного дрейфового расстояния в единицах характерного размера зеркала $d$-диаметра цилиндрического электрода), обусловленные решением $g=g(z)$, значительно (почти на порядок) превышают его значения, обусловленные решением $p=p(z)$, для всех режимов работы зеркала. Это связано с разной длиной пути ионов в зеркале и разной степенью торможения ионов на втором электроде зеркала.

Следует отметить, что безразмерная величина

$$
\bar{L}=L / d
$$

является функцией лишь осевого распределения электростатического потенциала.

С учетом (39) предельную разрешающую способность (11) можно записать в виде

$$
R=\frac{\bar{L} \cdot d}{\Delta z_{i}} .
$$

Как видно из (40), разрешающую способность массрефлектрона можно повысить либо увеличением $\bar{L}$ при малых $d$, либо увеличением $d$ при малых $\bar{L}$. В последнем случае мы приходим к росту габарита прибора в целом за счет увеличения его размера в поперечном направлении.

Ход траекторий частиц в трехэлектродном зеркале вращательной симметрии с времяпролетной фокусировкой по энергии до третьего порядка включительно для различных режимов его работы представлен на рис. $2-4$.

\section{Заключение}

Результаты исследований показывают, что для всех рассмотренных режимов работы электростатического зеркала вращательной симметрии значения удельного эффективного дрейфового расстояния значительно отличаются в зависимости от того, каким решением обусловлены эти результаты. Это дает возможность использования высокодисперсионных зеркал вращательной симметрии для повышения разрешающей способности времяпролетных масс-рефлектронов без увеличения их общих размеров, а также для создания более компактных приборов при сохранении их разрешающей способности.

\section{Финансирование работы}

Работа выполнена при финансовой поддержке гранта № АР05132483 по программе Министерства образования и науки Республики Казахстан.

\section{Конфликт интересов}

Авторы заявляют, что у них нет конфликта интересов. 


\section{Список литературы}

[1] Каратаев В.И., Мамырин Б.А., Шмикк Д.В. // ЖТФ. 1971. Т. 41. Вып. 7. С. $1498-1501$.

[2] Назаренко Л.М., Секунова Л.М., Якушев Е.М. А.С. SU 1 725289 A1, 1992.

[3] Явор М.И., Веренчиков А.Н. // Научное приборостроение. 2004. T. 14. № 2. C. 38-45.

[4] Бимурзаев С.Б., Дауменов Т., Секунова Л.М., Якушев Е.М. // ЖТФ. 1983. Т. 53. С. 524-528.

[5] Бимурзаев С.Б., Нурасылова О.С., Холодов И.А. // Вестник АУЭС. 2016. № 1. С. 36-43.

[6] Бимурзаев С.Б., Саутбекова 3.С. // Вестник АУЭС. 2018. № 4. C. $131-138$.

[7] Бимурзаев С.Б., Бимурзаева Р.С., Саркеев Б.Т. // РЭ. 1991. T. 36. C. $2186-2195$.

[8] Бобыкин Б.В., Невинный Ю.А., Якушев Е.М. // ЖТФ. 1975. T. 45 . C. $2368-2372$. 